\title{
Flavour chemical dynamics during fermentation of kombucha tea
}

\author{
Zhen-jun Zhao' ${ }^{1 *}$, Yu-cheng Sui', Hua-wei Wu², Cai-bi Zhou ${ }^{3}$, Xian-chun Hu', Jian Zhang ${ }^{1}$ \\ ${ }^{1}$ College of Horticulture and Landscape Architecture, Yangtze University, Jingzhou, Hubei 434025, PR China; ${ }^{2}$ College of Life Science, Yangtze \\ University, Jingzhou, Hubei 434025, PR China; ${ }^{3}$ Qiannan Normal University for Nationalities, Duyun, Guizhou 558000, PR China
}

\section{A B S T R A C T}

\begin{abstract}
Main physical and chemical properties, microbial population and flavour compounds of kombucha fermentation were dynamically analyzed. The results showed: the $\mathrm{pH}$ values decreased but total acidity increased in kombucha fermentation broth with fermentation time; the concentrations of tea polyphenols and free amino acids firstly increased in the initial stage and then decreased till the end of fermentation; total catechins and caffine in kombucha fermentation broth were degraded progressively with fermentation time. Only 21 volatile flavour compounds were identified in the initial kombucha fermentation broth but 56 volatile flavour compounds were identified in the $10 \mathrm{~d}$ fermentation. The largest group was acids among which there were 22 different types of acids compounds, accounting for up to $57.21 \%$ of all volatile flavour components. Bacteria were more abundant and diverse than yeasts in kombucha fermentation broth during fermentation. We separated and identified 8 main microbial communities in kombucha fermentation broth during kombucha fermentation; 6 bacteria belonged to Gluconacetobacter saccharivorans, Acetobacter sp., Gluconacetobacter sp., Gluconacetobacter europaeus, Acetobacter aceti and Lactobacillus fermentum, and 2 yeasts belonged to Saccharomyces cerevisiae and Arxula adeninivorans. According to the production of organic acids i.e., acetic acid, we envisaged that the predominant bacterial species identified were probably Acetobacter sp. and Acetobacter aceti after 5 days fermentation.
\end{abstract}

Keywords: Kombucha tea; Fermentation; Flavour components; GC-MS

\section{INTRODUCTION}

Kombucha tea is a valuable health beverage, originated in Asia country over two thousand years ago and then rapidly spread to Europe and the rest of the world (Dipti et al., 2003). It is produced by kombucha (a symbiotic association, also named "tea fungus") fermentation on sweetened black tea infusion. With many different names across the world, like Mu-Go in Russian, Kombuchaschwamm in Germany and Finkochinese in Italian (Jayabalan et al., 2014), this fermented beverage is also called "the ultimate health drink" because a large number of positive claims regard it as a prophylactic agent (Blanc et al.,1996; Jayabalan et al., 2014). It has various biological functions and brings beneficial effects to human health like anti-oxidant and anti-cancer (Jayabalan et al., 2011), helping in the treatment of metabolic diseases such as gastric ulcers (Banerjee et al., 2010), and high cholesterol (Yang et al., 2009); This tea is also claimed to have beneficial effects on mitigation of the pains and stress from malignant diseases (Screeramulu et al., 2000).
Fermentation is a key process for kombucha tea to develop its beneficial quality. Tea fungus is a symbiotic association, which plays a great role in the fermentation of Kombucha tea. Such symbiotic association (tea fungus) is generally considered to be composed primarily of various yeasts (such as Saccharomyces spp., Torulopsis spp., Pichia spp., Brettanomyces spp., and Zygosaccharomyces kombuchaensis) and acetic acid bacteria (including Acetobacter aceti and Gluconobacter oxydans). In addition, few reports confirmed that lactic acid bacteria (LAB) was often found in the Kombucha fermentation process and facilitated this fermentation process. However, tea fungus is a complex and dynamic microbial ecosystem and its composition changes with fermentation process (Chen et al., 2000; Marsh et al., 2014; Chakravorty et al., 2016). With the dynamic changes of microbial community, changes of chemical composition (including functional constituents and flavour components) of kombucha tea understandably occur during kombucha fermentation. During the past decades, research on kombucha was highly boosted and considerable scientific investigations of the beverage were

\footnotetext{
${ }^{*}$ Corresponding author:

Zhen-jun Zhao, College of Horticulture and Landscape Architecture, Yangtze University, Jingzhou, Hubei 434025, PR China

Fax:+86716 8060561. E-mail: 781179278@qq.com
} 
conducted under static conditions aiming to unearth the chemical nature behind beneficial effects on human health. Over hundred kinds of chemical composition including gluconic, acetic, lactic, usinic acid, citric and oxalic had been revealed in kombucha tea so far (Jayabalan et al.,2014; Zhao et al., 2010), which could be divided into ethanol, aldehydes, ketones, acids, esters, amino acids, etc. Although the degradation dynamics of functional components such as tea polyphenols and catechins have been reported in detail to explore the formation mechanisms of functional qualities of kombucha tea, very little information is available regarding dynamic changes for the flavour constituents of kombucha tea during its whole fermentation process. Since the flavour quality of kombucha tea as a beverage is important in its production process, it is necessary to carry out more extensive exploration on flavour chemical dynamics of kombucha tea.

In this study, we investigate the physicochemical properties (like $\mathrm{pH}$ values, total acidity, changes of microbial population, tea polyphenols compounds and catechin categories) and analyze systematically flavour chemical dynamics of kombucha fermented for different periods of time by methods such as GC-MS. Such an exploration may eventually allow the correlation of flavour chemical dynamics to the fermentation process, thus, improving flavour quality of kombucha tea.

\section{MATERIALS AND METHODS}

\section{Materials and chemicals}

For the fermentation, Kombucha starter culture were collected from local producer in Heilongjiang province, China, and maintained in our Tea Analysis Laboratory at Yangtze University. Raw Pu-erh tea samples used in this study were provided by Yiliang Tea Company in Yunnan province. The peptone, beef extract and Dichloran RoseBengal Chloramphenicol used for medium ingredients were purchased from Baiao Biological Technology Co.(Shanghai, China). Unless stated, All the chemicals and solvents were of analytical grade.

\section{Preparation of kombucha tea}

Kombucha fermentation medium was prepared in triplicate using $8 \mathrm{~g}$ of raw Pu-erh tea, $1 \mathrm{~L}$ of boiled tap water, $100 \mathrm{~g}$ sucrose. Tea were added to boiling tap water and allowed to infuse for about $10 \mathrm{~min}$ at $100^{\circ} \mathrm{C}$ after which the infusions were filtered through gauze to remove tea leaves. $100 \mathrm{~g}$ of sucrose were dissolved in hot tea soup $\left(60^{\circ} \mathrm{C}\right)$ and the preparation was cooled to room temperature. The sugared tea soup was filtered with microporous membrane $(0.45 \mu \mathrm{m})$ to remove bacterium and then distributed into $250 \mathrm{ml}$ flasks $(150 \mathrm{ml} /$ per flask) that had been previously sterilized at $121^{\circ} \mathrm{C}$ for $20 \mathrm{~min}$.
The cooled tea soup was inoculated with $20 \mathrm{ml}$ actively growing tea fungus. The flasks were carefully covered with a clean cloth and fastened quickly. The fermentation was carried out at constant temperature of $28^{\circ} \mathrm{C}$ for about 14 days. Samples were collected from the flasks at different incubation time at $0,3,5,7,10$ and 14 days to measure fermentation-related indicators, including $\mathrm{pH}$, total acidity, microbial population, tea polyphenols compounds, catechin categories during fermentation of kombucha. Also, flavor chemical components generating in metabolism process of komucha were analyzed systematically using the methods of GC-MS. Each flask was sampled only once to avoid potential microbial contamination, and all analyses were carried out in triplicate.

\section{Microbiological analysis}

The Microbial Population of fermented liquid was enumerated from different incubation time at $0,3,5$, 7, 10 and 14 days using the method of dilution plating as described previously by Pitt et al.(1997). Ten $\mathrm{ml}$ of each sample were added to $90 \mathrm{ml}$ of $0.1 \%$ peptone water. This mixture was then shaken on a rotary shaker for approximately $15 \mathrm{~min}$. A decimal dilution series was prepared with sterile water, and $0.1 \mathrm{ml}$ of the appropriately diluted suspension was spread on the surface of the medium (Dichloran Rose-Bengal Chloramphenicol Agar medium for yeast and beef extract peptone medium for bacterium). All plates were incubated at $25 \sim 30^{\circ} \mathrm{C}$ for up to 7 days, with bacterium being enumerated after 3 days and yeasts after 5-7 days. Single and well-isolated colonies were picked from the media and were cultured on the medium (Czapek yeast extract media for yeast and beef extract peptone medium for bacterium) for subsequent characterization and taxonomic identification procedures.

Pure colonies were sub-cultered into $50 \mathrm{~mL}$ liquid media (Czapek yeast extract media for yeast and beef extract peptone medium for bacterium) (Pitt and Hocking, 1997) in $150 \mathrm{~mL}$ erlenmeyer flasks and incubated in an orbital shaker at $100 \mathrm{rpm}$ at $25^{\circ} \mathrm{C}$ to grow for 7 days or more. The fresh biomass was harvested for DNA extraction and analysis of ITS or 16S rDNA sequence. The universal primers ITS1 (5'-TCCGTAGGTGAACCTGCCG-3'), ITS4 (5'-TCCTCCGCTTATT GATATGC-3') for yeasts and 27F (5'-AGAGTTGATCCTGGCTCATCAG-3'), 1504R (5'-AAGG AGGTGATCCAGCCGCA-3') for bacterium (synthesized by TaKaRa Biotechnology Co., Ltd. Dalian, China) were used for PCR reaction to amplify ITS and $16 \mathrm{~S}$ rDNA sequence, by means of the gained DNA as the templates. Amplifications products after purification were sent to sequencing facility (by Invitrogen Corp.). Sequences were used in an ungapped BLAST search of the GenBank database for the microbial identification. 


\section{Physico-chemical analysis}

Values of $\mathrm{pH}$ were measured using an electronic $\mathrm{pH}$ meter (Jenco, Shanghai, China). Total acidity was determined by titration with a standard solution of sodium hydroxide and phenolphthalein as indicator (GB/T12456-90).

The ferrous tartrate spectrophotometric method was applied to measure total polyphenols of fermentation liquid (Liao et al. 2002). Three independent measurements were done for each sample in order to check the reproducibility of the obtained data.

Constituents and concentrations of Catechins in fermentation liquid were determined by methods of highperformance liquid chromatography (HPLC) described by Xu et al. (2005).

Total content of free amino acids in the fermented liquid of Kombucha was determined according to the method as previously described by Jiang et al.(2007).

\section{Determining the volatile flavour components present in kombucha fermentation broth at different stages of fermentation}

All samples were analyzed in duplicate with the same procedure. The protocol employed for extraction of volatile chemical components in the Kombucha tea broth was similar to that reported by José et al. (2001). One milliliter of fermented liquid was mixed with $0.3 \mathrm{~g} \mathrm{NaCl}$ and $0.17 \mu \mathrm{g}$ of 2 -nonanone, as an internal standard in a $4 \mathrm{ml}$ vial. The mixture was shaken for $2 \mathrm{~min}$, and mixed solution was heated with water bath at $30^{\circ} \mathrm{C}$.After $10 \mathrm{~min}$ of vial equilibration time, the fiber holder plunger was depressed to insert ca. $1 \mathrm{~cm}$ of the coated fused-silica fiber into the head-space sample for $30 \mathrm{~min}$. Then the fiber was withdrawn out of the sample and inserted into the injection port of a gas chromatographic mass spectrometer (GC-MS) (The Shimadzu in Japan) for the analysis of volatile flavour components.

GC separation was accomplished on Rtx-SMS column $(30 \mathrm{~m} \times 0.25 \mathrm{~mm}$ i.d., $0.25 \mu \mathrm{m}$ particle size $)$. The inlet temperature was set at $260^{\circ} \mathrm{C}$. The oven temperature program was started at an initial temperature of $80^{\circ} \mathrm{C}$, lasting for $3.0 \mathrm{~min}$, then increased to $260^{\circ} \mathrm{C}$ at $10{ }^{\circ} \mathrm{C} / \mathrm{min}$ and retained for $3.0 \mathrm{~min}$. Samples were injected in the split mode $(1 / 10)$, using helium as carrier gas $(99.9 \%, 1 \mathrm{~mL} / \mathrm{min})$. In the MS system, both the ion source temperature and inlet temperature were $230^{\circ} \mathrm{C}$, scanned area was 5-550amu. Identification of unknown volatiles was based on comparison of their mass spectra with those from NIST 05 and NIST 05s MS libraries. The quality of each compound was determined using the area normalization method (José et al. 2001).

\section{Data analysis}

All chemical analyses and measures of fermentation liquid samples were performed in triplicate $(n=3)$ and values were describled as an average value in this paper. Univariate treatments of data were carried out by analysis of variance (ANOVA), using "Microsoft Office Excel 2007” software program.

\section{RESULTS}

\section{Variation in taste properties during fermentation of kombucha \\ Differences in $\mathrm{pH}$ values, total acidity, tea polyphenols and total free amino acids concentration during fermentation}

The $\mathrm{pH}$ values in fermentation broth decreased with the fermentation of kombucha (Fig. 1). From the initiation of kombucha fermentation during $0-10 \mathrm{~d}$, the $\mathrm{pH}$ value declined quickly from 6.71 to 2.91 and decreased to the minimum value (about 2.9-3.0) at the $10^{\text {th }}$ day, but thereafter it increased slowly from 2.91 to 3.74 at the $14^{\text {th }}$ day. There was a negative correlation between $\mathrm{pH}$ value and total acidity, meaning that acidity increased with the decrease of $\mathrm{pH}$ value (Fig. 1). Increase of acidity showed that microorganisms produced many organic acid substances during fermentation of kombucha. The initial concentration of acids was at the lowest level; less than 0.09 . As the fermentation proceeded, changes of acidity could be divided into two stages: the rapid increase stage $(0-10 \mathrm{~d})$ and the slow decrease stage (from 10-14 d); the acidity increased quickly from 0.09 to 2.59 - the peak value during the first stage.

Content of total tea polyphenols and free amino acids in kombucha broth during fermentation were estimated by means of spectrophotometry. The concentrations of total tea polyphenols and free amino acids, varying with fermentation time are shown in Fig.2. It was found that fluctuation changes of total tea polyphenols and

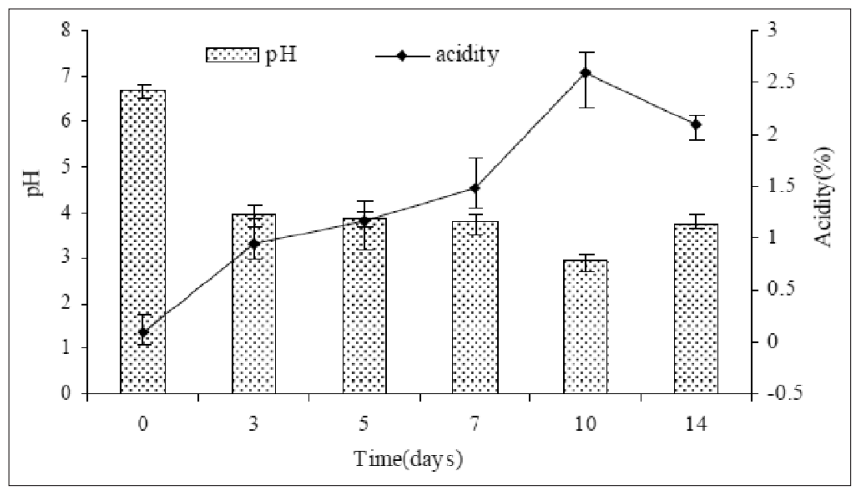

Fig 1. Differences in $\mathrm{pH}$ and total acidity of kombucha broth during fermentation 
free amino acids in kombucha broth showed an almost identical trend during the whole fermentation process. The concentrations of tea polyphenols decreased slowly up to the $3^{\text {rd }}$ day of kombucha fermentation and increased from $1.89 \mathrm{mg} / \mathrm{mL}$ at the $3^{\text {rd }}$ day to $2.56 \mathrm{mg} / \mathrm{mL}$ at the $5^{\text {th }}$ day, but thereafter reduced to $1.75 \mathrm{mg} / \mathrm{mL}$ at the $10^{\text {th }}$ day. By the end of fermentation $(14 \mathrm{~d})$, the content of tea polyphenols was $1.86 \mathrm{mg} / \mathrm{mL}$. The quantity of free amino acids in kombucha broth firstly decreased slightly, and then increased significantly to the peak of $0.73 \mathrm{mg} / \mathrm{mL}$ at the $7^{\text {th }}$ day. Later the content of free amino acids reduced and remained at $0.58 \mathrm{mg} / \mathrm{mL}$ by the end of fermentation (14 d).

\section{Changes of catechins and caffine in kombucha fermentation broth at different stages of fermentation}

Six different catechins and caffine were evaluated in kombucha fermentation broth at different fermentation stages using HPLC methods. The measured results are shown in table 1 . Across the span of 14 days kombucha fermentation, total catechins and caffine were degraded progressively with fermentation time in kombucha fermentation broth; and the relative concentration of total catechins and caffine in kombucha fermentation broth, compared to the initial stage $(0$ d), decreased by $43.7 \%, 19.7 \%$ respectively. Six different catechins in kombucha fermentation broth

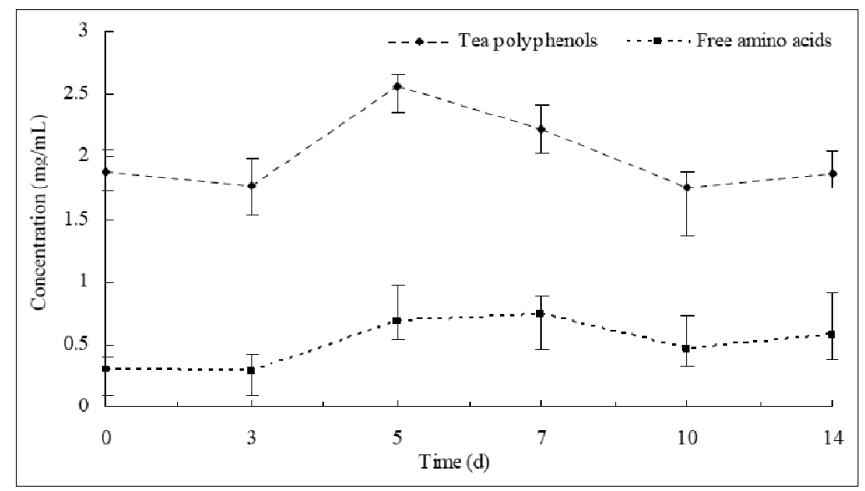

Fig 2. Differences in tea polyphenols and free amino acids of kombucha broth during fermentation could be divided into ester type catechins such as (-)-Epicatechin gallate (ECG), (-)-Epigallocatechin gallate (EGCG), (-)-Gallocatechin gallate (GCG), and non-ester type catechins including $(-)$-Epicatechin (EC), $(+)$-Catechin (C) and (-)-Epigallocatechin(EGC). During kombucha fermentation, the concentration of ester type catechins(including EGCG, ECG and GCG)decreased constantly. For example, the concentration of EGCG reduced from $0.307 \mathrm{mg} / \mathrm{mL}$ at $0 \mathrm{~d}$ to $0.135 \mathrm{mg} / \mathrm{mL}$ at the end-fermentation stage, GCG reduced from $0.153 \mathrm{mg} / \mathrm{mL}$ at $0 \mathrm{~d}$ to $0.075 \mathrm{mg} / \mathrm{mL}$ at the end-fermentation stage and ECG reduced from $0.079 \mathrm{mg} / \mathrm{mL}$ at $0 \mathrm{~d}$ to $0.040 \mathrm{mg} / \mathrm{mL}$ at the end-fermentation stage. However, non-ester type catechins contents in kombucha fermentation broth had gained a slight increase during 14 days kombucha fermentation, for example, the concentration of EGC increased from $0.031 \mathrm{mg} / \mathrm{mL}$ at $0 \mathrm{~d}$ to $0.041 \mathrm{mg} / \mathrm{mL}$ at the end-fermentation stage.

\section{Differences in microbial population of kombucha fermentation broth during fermentation}

Microbial population of kombucha fermentation broth was composed of yeasts and bacteria. The total number of yeasts and bacteria at different stages of kombucha fermentation is shown in Table 2. The initial number of yeasts and bacteria in sugared raw $\mathrm{Pu}$-erh tea broth was at the level of $7.43 \times 10^{3} \mathrm{CFU} / \mathrm{mL}, 1.41 \times 10^{4} \mathrm{CFU} / \mathrm{mL}$ respectively. The total count of bacteria increased quickly to the maximum of $2.70 \times 10^{9} \mathrm{CFU} / \mathrm{mL}$ at the $5^{\text {th }}$ day, and decreased gradually to a lower level of $1.41 \times 10^{6} \mathrm{CFU} /$ $\mathrm{mL}$ at $14^{\text {th }}$ day. The total count of yeasts increased to the peak of $6.01 \times 10^{6} \mathrm{CFU} / \mathrm{mL}$ at $7^{\text {th }}$ day, but thereafter it remained at a level of $7.49 \times 10^{5} \mathrm{CFU} / \mathrm{mL}$ by the end of fermentation.

A total of 15 microbial strains (including 5 yeasts and 10 bacterial strains) were isolated from the kombucha fermentation broth through dilution plating. After a preliminary morphological identification, 8 isolates (including 2 yeasts and 6 bacterial isolates) were successfully applied to be further identified at species level by means of molecular methods. The microbial

Table 1: Dynamic changes of tea catechins and caffine in kombucha broth during fermentation $(\mathrm{mg} / \mathrm{mL})$

\begin{tabular}{|c|c|c|c|c|c|c|}
\hline \multirow[t]{2}{*}{ Constituents } & \multicolumn{6}{|c|}{ Contents } \\
\hline & Od & $3 d$ & $5 d$ & $7 d$ & $10 d$ & $14 d$ \\
\hline Total catechins & $0.572 \pm 0.008$ & $0.483 \pm 0.001$ & $0.415 \pm 0.011$ & $0.421 \pm 0.015$ & $0.394 \pm 0.016$ & $0.322 \pm 0.016$ \\
\hline EGC & $0.031 \pm 0.005$ & $0.037 \pm 0.013$ & $0.041 \pm 0.007$ & $0.042 \pm 0.003$ & $0.047 \pm 0.001$ & $0.041 \pm 0.001$ \\
\hline DL-C & $0.016 \pm 0.027$ & $0.024 \pm 0.001$ & $0.038 \pm 0.002$ & $0.037 \pm 0.001$ & $0.036 \pm 0.000$ & $0.031 \pm 0.000$ \\
\hline EC & $0.011 \pm 0.002$ & $0.019 \pm 0.019$ & $0.018 \pm 0.006$ & $0.025 \pm 0.004$ & $0.021 \pm 0.003$ & $0.027 \pm 0.006$ \\
\hline EGCG & $0.307 \pm 0.001$ & $0.226 \pm 0.002$ & $0.216 \pm 0.016$ & $0.241 \pm 0.011$ & $0.210 \pm 0.010$ & $0.135 \pm 0.002$ \\
\hline GCG & $0.153 \pm 0.014$ & $0.141 \pm 0.022$ & $0.106 \pm 0.000$ & $0.107 \pm 0.007$ & $0.098 \pm 0.001$ & $0.075 \pm 0.001$ \\
\hline ECG & $0.079 \pm 0.012$ & $0.064 \pm 0.017$ & $0.066 \pm 0.005$ & $0.059 \pm 0.001$ & $0.042 \pm 0.008$ & $0.040 \pm 0.009$ \\
\hline Caffine & $0.121 \pm 0.002$ & $0.120 \pm 0.009$ & $0.101 \pm 0.008$ & $0.110 \pm 0.003$ & $0.110 \pm 0.007$ & $0.097 \pm 0.012$ \\
\hline
\end{tabular}


DNA sequence (yeasts for ITS and bacteria for $16 \mathrm{~S}$ rDNA) were amplified and the purified PCR products were sequenced by sequencing facility. PCR amplification products of ITS or $16 \mathrm{~S}$ rDNA of microbial communities of kombucha fermentation broth are shown in Fig.3. Among the identified microbial strains, 2 species belonged to yeast, ie. Saccharomyces cerevisiae and Arxula adeninivorans; 6 belonged to bacteria: Gluconacetobacter saccharivorans, Acetobacter sp., Gluconacetobacter sp., Gluconacetobacter europaeus, Acetobacter aceti and Lactobacillus fermentum (shown in Table 3).

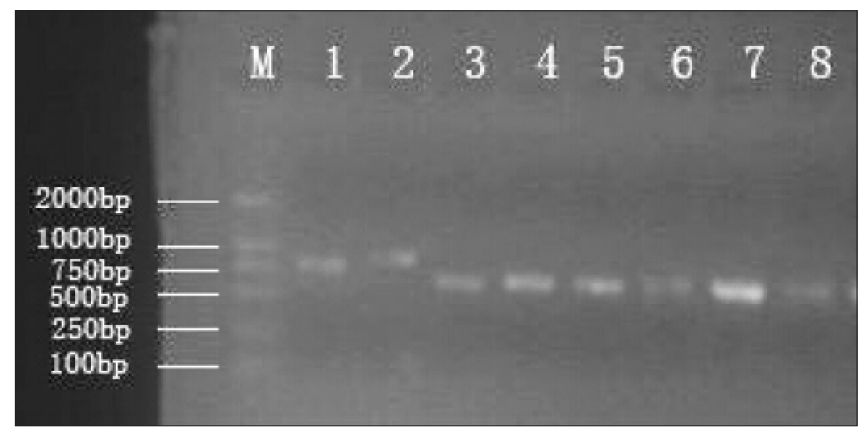

Fig 3. PCR amplification of ITS or 16S rDNA of microbial communities present in the kombucha fermentation broth
Differences in volatile flavour components of kombucha fermentation broth during fermentation

The volatile flavour components in kombucha fermentation broth during fermentation were extracted by HS-SPME and then analyzed by GC-MS. The measurement results of volatile flavour are shown in Table 4. The composition and concentration of volatile flavour matters measured varied with fermentation stages of kombucha. There were $21,46,52,57,56$ and 53 different volatile flavour components identified during fermentation time ie. $0 \mathrm{~d}, 3 \mathrm{~d}$, $5 \mathrm{~d}, 7 \mathrm{~d}, 10 \mathrm{~d}$ and $14 \mathrm{~d}$ respectively. In general, these volatile flavour components could be divided into 7 different types, such as alcohols, aldehydes, ketones, acids, esters and benzenoids. The volatile flavour components (including composition and content) were at a state of dynamic change throughout the fermentation process. Some volatile flavour components increased and others decreased, which impacted the dynamic changes of flavour characterics of kombucha fermentation broth over the whole fermentation period.

21 volatile flavour components, identified in kombucha broth of $0 \mathrm{~d}$ fermentation, belonged to alcohols, aldehydes, ketones, acids, esters, benzenoids. Most of them came from

Table 2: Changes of microbial population during the fermentation of Kombucha in sugared tea soup

\begin{tabular}{|c|c|c|c|}
\hline \multicolumn{2}{|c|}{ Fermentation time } & \multirow{2}{*}{$\frac{\text { Bacteria }}{1.41 \pm 0.77 \times 10^{4}}$} & \multirow{2}{*}{$\begin{array}{l}\text { Yeasts } \\
7.43 \pm 0.35 \times 10^{3}\end{array}$} \\
\hline $0 \mathrm{~d}$ & Total number of microbial population (CFU/mL) & & \\
\hline & Microbial species with higher isolation frequency & $\begin{array}{l}\text { Gluconobacter sp. } \\
\text { Gluconacetobacter europaeus }\end{array}$ & Saccharomyces cerevisiae \\
\hline \multirow[t]{2}{*}{$3 d$} & Total number of microbial population (CFU/mL) & $1.35 \pm 0.27 \times 10^{9}$ & $3.19 \pm 0.21 \times 10^{4}$ \\
\hline & Microbial species with higher isolation frequency & $\begin{array}{l}\text { Gluconacetobacter europaeus, } \\
\text { Acetobacter aceti }\end{array}$ & Saccharomyces cerevisiae \\
\hline \multirow[t]{2}{*}{$5 d$} & Total number of microbial population (CFU/mL) & $2.70 \pm 0.16 \times 10^{9}$ & $2.37 \pm 0.26 \times 10^{6}$ \\
\hline & Microbial species with higher isolation frequency & $\begin{array}{l}\text { Gluconacetobacter europaeus, } \\
\text { Acetobacter aceti }\end{array}$ & $\begin{array}{l}\text { Saccharomyces cerevisiae, } \\
\text { Arxula adeninivorans }\end{array}$ \\
\hline \multirow[t]{2}{*}{$7 d$} & Total number of microbial population (CFU/mL) & $7.09 \pm 0.14 \times 10^{8}$ & $6.01 \pm 0.89 \times 10^{6}$ \\
\hline & Microbial species with higher isolation frequency & Acetobacter aceti & $\begin{array}{l}\text { Saccharomyces cerevisiae, } \\
\text { Arxula adeninivorans }\end{array}$ \\
\hline \multirow[t]{2}{*}{$10 \mathrm{~d}$} & Total number of microbial population (CFU/mL) & $6.93 \pm 0.33 \times 10^{6}$ & $7.52 \pm 0.57 \times 10^{5}$ \\
\hline & Microbial species with higher isolation frequency & Acetobacter aceti & Arxula adeninivorans \\
\hline \multirow[t]{2}{*}{$14 d$} & Total number of microbial population (CFU/mL) & $1.48 \pm 0.21 \times 10^{6}$ & $6.49 \pm 0.17 \times 10^{5}$ \\
\hline & Microbial species with higher isolation frequency & Acetobacter aceti & Arxula adeninivorans \\
\hline
\end{tabular}

Table 3: Identification of microbial communities (by 16S rDNA or ITS) present in kombucha fermentation broth, based on BLAST comparison in GenBank

\begin{tabular}{lcclc}
\hline No. & $\begin{array}{c}\text { Fragment length } \\
\text { of ITS (bp) }\end{array}$ & $\begin{array}{c}\text { Sequence } \\
\text { similarity (\%) }\end{array}$ & Alignment result & $\begin{array}{c}\text { GenBank } \\
\text { accession no. }\end{array}$ \\
\hline 1 & 780 & $100 \%$ & Saccharomyces cerevisiae & $\mathrm{KY} 433311$ \\
2 & 722 & $99 \%$ & Arxula adeninivorans & $\mathrm{KY} 433312$ \\
3 & 518 & $98 \%$ & Gluconacetobacter saccharivorans & $\mathrm{KY} 468827$ \\
4 & 480 & $99 \%$ & Acetobacter sp & $\mathrm{KY} 468828$ \\
5 & 387 & $97 \%$ & Gluconobacter sp. & $\mathrm{KY} 468829$ \\
6 & 460 & Gluconacetobacter europaeus & $\mathrm{KY} 468830$ \\
7 & 426 & Acetobacter aceti & $\mathrm{KY} 468831$ \\
8 & 435 & $97 \%$ & Lactobacillus fermentum & $\mathrm{KY} 468832$ \\
\hline${ }^{*}$ GenBank (NCBI, National Centre for Biotechnology Informatio, Bethesda, MD) & &
\end{tabular}


Table 4: Volatile flavour compound peak area: Internal standard peak area ratio of kombucha fermentation broth

\begin{tabular}{|c|c|c|c|c|c|c|c|c|}
\hline \multirow[t]{2}{*}{ No. } & \multirow[t]{2}{*}{ Category } & \multirow[t]{2}{*}{ Compounds } & \multicolumn{6}{|c|}{ Relative content (peak area) } \\
\hline & & & od & $3 d$ & $5 d$ & $7 d$ & $10 \mathrm{~d}$ & $14 d$ \\
\hline 1 & Alcohols & 2,6-dimethyl-4-ethyl-4-heptanol & 0.64 & 1.69 & 2.91 & 0.71 & 0.58 & 0.29 \\
\hline 2 & & 2,7-dimethyl-4-octene-2,7-diol & $\operatorname{tr}$ & $\operatorname{tr}$ & 0.12 & 2.78 & 0.23 & 0.02 \\
\hline 3 & & 2-ethyl-2-methyl-tridecanol & nd & 0.28 & 0.96 & 0.10 & 0.70 & 0.43 \\
\hline 4 & & 3,5-dimethyl cyclohexanol & nd & nd & 0.19 & 0.99 & 0.87 & 0.71 \\
\hline 5 & & 5-methyl-2-piperazinyl methanol & nd & 0.37 & 1.96 & 1.56 & 1.05 & 0.78 \\
\hline 6 & & phenethyl alcohol & 2.53 & 1.20 & 1.49 & 1.08 & nd & nd \\
\hline 7 & & 3,7-dimethyl-1,6-octadiene-3-alcohol & nd & 0.48 & 0.72 & 0.63 & 0.41 & nd \\
\hline 8 & & 1-ethyl-2-methyl cyclohexanol & nd & 1.42 & 1.79 & 1.51 & 0.49 & $\operatorname{tr}$ \\
\hline 9 & & 3-methyl-2-isopropyl cyclohexanol & nd & 0.33 & 1.37 & 0.66 & 0.61 & 0.49 \\
\hline 10 & & 2-isopropyl-5-methyl-1-heptanol & 0.17 & 0.37 & 1.92 & 0.73 & 0.32 & 0.25 \\
\hline 11 & & linalool & 6.15 & 2.11 & 1.13 & 0.62 & 0.61 & 0.50 \\
\hline 12 & & terpineol & 2.03 & 1.70 & 0.66 & 0.61 & 0.49 & 0.44 \\
\hline 13 & & 2,5-dimethyl-4-hexene-3-alcohol & 0.13 & 0.79 & 2.78 & 1.30 & 0.78 & 0.31 \\
\hline 14 & & 2-methyl decanol & 1.10 & 0.31 & 1.52 & 1.07 & nd & nd \\
\hline 15 & & 2-hexyl-1-decanol & 0.32 & 0.35 & 2.77 & 0.11 & $\operatorname{tr}$ & $\operatorname{tr}$ \\
\hline 16 & & 5-methyl-2-isopropyl hexanol & 0.52 & 0.75 & 2.78 & 1.03 & nd & nd \\
\hline 17 & Aldehydes & Z-7-hexadecenal & 1.01 & 1.33 & 1.67 & 0.36 & $\operatorname{tr}$ & $\operatorname{tr}$ \\
\hline 18 & & furfural & 1.14 & 0.27 & 0.24 & 0.23 & $\operatorname{tr}$ & $\operatorname{tr}$ \\
\hline 19 & & benzeneacetaldehyde & 7.46 & 3.01 & 1.21 & 1.15 & $\operatorname{tr}$ & $\operatorname{tr}$ \\
\hline 20 & & heptaldehyde & 5.86 & 3.34 & 3.25 & 1.68 & 0.38 & nd \\
\hline 21 & & 2,4-dimethyl benzaldehyde & 3.13 & 1.97 & 1.38 & 0.92 & 0.83 & 0.71 \\
\hline 22 & & nonanal & 1.84 & 1.62 & 1.19 & 1.01 & $\operatorname{tr}$ & nd \\
\hline 23 & Ketones & 2-heptanone & 1.72 & 0.91 & 0.73 & 0.60 & 0.55 & 0.52 \\
\hline 24 & & phenylacetone & 0.56 & 0.73 & 0.68 & 1.43 & nd & nd \\
\hline 25 & & methyl octyl ketone & 3.32 & 2.61 & 2.75 & 2.30 & 2.15 & 2.19 \\
\hline 26 & Acids & acetic acid & 0.30 & 1.79 & 8.12 & 19.53 & 21.64 & 23.90 \\
\hline 27 & & phenylacetic acid & 2.32 & 2.72 & 2.30 & 2.39 & 3.35 & 1.31 \\
\hline 28 & & 2-methyl succinic acid & nd & 0.01 & 0.41 & 1.14 & 2.78 & 1.88 \\
\hline 29 & & malonic acid & nd & nd & nd & 0.39 & 0.58 & 0.29 \\
\hline 30 & & 2-hydroxy malonic acid & nd & nd & 0.19 & 0.27 & 0.51 & 0.63 \\
\hline 31 & & propanoic acid & nd & nd & nd & $\operatorname{tr}$ & 1.26 & 1.04 \\
\hline 32 & & succinic acid & 0.47 & 0.55 & 0.58 & 0.74 & 2.48 & 2.31 \\
\hline 33 & & benzeneacetic acid & nd & 0.10 & 1.27 & 1.38 & 1.33 & 1.35 \\
\hline 34 & & oxalic acid & 0.93 & 0.95 & 1.09 & 1.63 & 1.75 & 1.52 \\
\hline 35 & & 2-oxo-malonic acid & nd & nd & $\operatorname{tr}$ & $\operatorname{tr}$ & 0.46 & 0.48 \\
\hline 36 & & L-lactate & nd & 0.37 & 2.16 & 2.59 & 2.70 & 2.11 \\
\hline 37 & & 2-oxo-glutaric acid & nd & 0.26 & 0.79 & 0.91 & 1.16 & 1.36 \\
\hline 38 & & butanoic acid & nd & nd & 0.06 & 2.51 & 3.41 & 3.30 \\
\hline 39 & & 2-oxovaleric acid & nd & nd & nd & 0.31 & 0.56 & 0.66 \\
\hline 40 & & 4-oxovaleric acid & nd & nd & nd & nd & 0.73 & 0.79 \\
\hline 41 & & citric acid & 0.19 & 0.47 & 1.77 & 2.59 & 3.21 & 3.36 \\
\hline 42 & & 2-oxocaproic acid & nd & nd & 0.51 & 0.22 & 0.25 & 0.27 \\
\hline 43 & & 5-hydroxy-3,4,4-trimethacrylic acid & $\operatorname{tr}$ & 0.61 & 0.49 & 0.29 & 0.10 & 0.09 \\
\hline 44 & & 2-hydroxy-3-methbutyric acid & $\operatorname{tr}$ & 0.94 & 1.40 & 1.94 & 1.48 & 1.25 \\
\hline 45 & & D-glucuronic acid & nd & nd & nd & 0.48 & 1.29 & 1.36 \\
\hline 46 & & nonahexacontanoic acid & nd & 0.56 & 0.82 & 0.88 & 0.90 & 0.93 \\
\hline 47 & & 2-methyl-3-hydroxy valeric acid & nd & nd & nd & 0.49 & 0.36 & 0.40 \\
\hline 48 & Esters & 3-methyl propionic acid-2-acrylic ester & nd & 0.67 & 0.77 & 1.61 & 1.51 & 0.94 \\
\hline 49 & & methoxyacetic acid-4-hexadecane ester & nd & 0.43 & 0.84 & 1.12 & 1.30 & 1.37 \\
\hline 50 & & diisononyl phthalate & nd & nd & nd & nd & 0.92 & 0.28 \\
\hline 51 & & 2,6-hexadecyl-1(+)-ascorbic ester & nd & 0.50 & 0.85 & 1.57 & 3.36 & 4.32 \\
\hline 52 & & octadecyl acetate & 1.11 & 1.26 & 1.32 & 0.93 & 0.90 & 0.97 \\
\hline 53 & & isobornyl thiocyanoacetate & nd & 0.24 & 0.49 & 0.78 & 0.71 & 0.75 \\
\hline
\end{tabular}


Table 4: (Continued)

\begin{tabular}{|c|c|c|c|c|c|c|c|c|}
\hline \multirow[t]{2}{*}{ No. } & \multirow[t]{2}{*}{ Category } & \multirow[t]{2}{*}{ Compounds } & \multicolumn{6}{|c|}{ Relative content (peak area) } \\
\hline & & & Od & 3d & $5 d$ & $7 d$ & $10 \mathrm{~d}$ & $14 d$ \\
\hline 54 & Benzenoids & 1,3-dimethyl benene & 0.42 & 0.37 & 0.33 & 0.32 & 0.33 & 0.31 \\
\hline 55 & & 1,2,4-trimethoxybenzene & 1.20 & 1.27 & 1.25 & 1.68 & 1.62 & 1.56 \\
\hline 56 & & 1,2,3-trimethoxybenzene & 1.17 & 1.16 & 1.19 & 1.14 & 1.05 & 1.03 \\
\hline 57 & Others & decyl ether & nd & 0.41 & 0.53 & 0.75 & 0.93 & 1.12 \\
\hline 58 & & heptane & nd & nd & nd & nd & 0.33 & 0.71 \\
\hline 59 & & undecane & nd & nd & 0.36 & 0.76 & 0.83 & 0.89 \\
\hline 60 & & dodecane & 1.31 & 1.39 & 1.87 & 1.91 & 1.90 & 1.96 \\
\hline
\end{tabular}

nd means "not detected"; tr means "trace".

flavour components of raw Pu-erh tea itself. The maximum content in starting fermentation broth was aldehydes (including 6 different types of aldehydes) accounting for up to $18.60 \%$ of all volatile flavour components. The second highest was alcohols of which there were 9 different types sharing $13.59 \%$ of all volatile flavour components. Specifically it was found that the top five components were benzeneacetaldehyde, linalool, heptaldehyde, methyl octyl ketone and 2,4-dimethyl benzaldehyde, occupying $7.46 \%, 6.15 \%, 5.86 \%, 3.32 \%, 3.13 \%$ of all volatile flavour components in starting kombucha fermentation broth at $0 \mathrm{~d}$ fermentation.

46 volatile flavour components, identified in kombucha broth of $3 \mathrm{~d}$ fermentation, were alcohols, aldehydes, ketones, acids, esters, benzenoids and other compounds. The number of alcohol compounds identified and the concentration of acid compounds increased significantly during this fermentation period, compared with those of starting fermentation broth of $0 \mathrm{~d}$ fermentation. The largest group was alcohols among which there were 15 different types of alcohol compounds, accounting for up to $13.15 \%$ of all volatile flavour components. The variety with the second highest content in $3 \mathrm{~d}$ fermentation broth was acids (including 12 different types of acid compounds), sharing $12.04 \%$ of all volatile flavour components. As for individual volatile flavour compounds, the top five components were heptaldehyde, benzeneacetaldehyde, phenylacetic acid, methyl octyl ketone and linalool, occupying $3.34 \%, 3.01 \%, 2.72 \%, 2.61 \%$ and $2.11 \%$ of all volatile flavour components in kombucha fermentation broth of $3 \mathrm{~d}$ fermentation.

52 volatile flavour components were identified in kombucha broth of $5 \mathrm{~d}$ kombucha fermentation. During this fermentation period, the concentrations of alcohols and acids compounds increased continuously. The largest group was still alcohols among which there were 16 different types of alcohol compounds, accounting for up to $26.68 \%$ of all volatile flavour components. The variety with the second highest content in $5 \mathrm{~d}$ fermentation broth was acids (including 15 different types of acid compounds), sharing $23.22 \%$ of all volatile flavour components.
At the $7^{\text {th }}$ day of kombucha fermentation, 57 volatile flavour components were identified in kombucha broth. During this fermentation period, the content of alcohol compounds decreased while the concentration of acid compounds increased significantly. The top groups of volatile flavour components were still acids, alcohols and aldehydes compounds, accounting for up to $40.52,17.39 \%$ and $11.34 \%$ of all volatile flavour components respectively.

56 volatile flavour components were identified in kombucha broth of $10 \mathrm{~d}$ fermentation. The component and content of alcohol and aldehydes compounds decreased significantly and acids compounds increased continuously during this fermentation period. The largest group was acids among which there were 22 different types of acids compounds, accounting for up to $57.21 \%$ of all volatile flavour components. The top five acids components were acetic acid, butanoic acid, phenylacetic acid, citric acid and 2-methyl succinic acid, sharing 21.64\%, 3.41\%, 3.35\%, $3.21 \%$ and $2.78 \%$ of all volatile flavour components in kombucha fermentation broth of $10 \mathrm{~d}$ fermentation.

53 volatile flavour components were identified in kombucha broth of $14 \mathrm{~d}$ fermentation, which were mainly consisted of acids and esters compounds. The content of alcohols compounds decreased while acids compounds increased continuously during this fermentation period. The acids compounds (including 22 different types of acids compounds) shared $57.62 \%$ of all volatile flavour components. The top five acids components were acetic acid, citric acid, butanoic acid, succinic acid, and L-lactate, sharing $23.90 \%, 3.36 \%, 3.30 \%, 2.31 \%$ and $2.11 \%$ of all volatile flavour components in kombucha fermentation broth of $14 \mathrm{~d}$ fermentation by the end of kombucha fermentation.

\section{DISSCUSSION}

As a popular health beverage around the world, kombucha tea is recognized for its focus not just on health functions but also its flavour quality. This beverage is accepted because it has unique flavour of sweet, sour and refreshing, 
which results from characteristic chemical compounds (Kallel et al., 2012). The flavour chemical compounds of kombucha can be divided into three categories according to its origins: tea constituents and its derived ingredients, sugar substrates and extensive metabolic products produced by tea fungus during fermentation.

Tea polyphenols are one group of characteristic components, which account for $1.87 \mathrm{mg} / \mathrm{mL}$ in the sugared tea broth at unfermented stage $(0 \mathrm{~d})$. They are considered as the major contributor to the puckering astringency and bitterness taste. In the present study, the content of total tea polyphenols increased slightly at the beginning of fermentation (0-5 d) and then decreased hereafter, which was similar to the microbial diversity change trend during kombucha fermentation. It was thought that the increase in total tea polyphenols content in kombucha fermentation broth in the initial stage of fermentation (from $0 \mathrm{~d}$ to $5 \mathrm{~d}$ ) might be due to the decomposition of macromolecular polyphenols to small molecules under the effect of complex enzyme provided by bacteria and yeasts. This, in turn, caused the increase of total polyphenols (Jayabalan et al., 2007; Chakravorty et al., 2016). And the decrease of total tea polyphenols content in kombucha fermentation broth in later period of fermentation (from 5-14 d) might be the reason for the oxidation of tea polyphenols under oxygen free radical liberated by microorganisms especially in lower $\mathrm{pH}$ value condition (Wang et al., 2015). Consistent degradation was observed for total catechins throughout the fermentation process of kombucha (Jayabalan et al., 2007). Dynamic analysis on content of six epicatechin isomers during kombucha fermentation demonstrated that ester type catechins(including EGCG_ECG and GCG)decreased constantly and non-ester type catechins(including EC, C and EGC),contents increased slightly. It was assumed that ester type catechins were converted to their corresponding catechins under the effects of enzymes liberated by complex tea fungus in the acidic environment (Jayabalan et al., 2014).

Amino acids and caffine are important flavour substances, which are considered as the potential contributors to the refreshing and bitterness taste of tea respectively. Although no other nitrogen source was added to initial fermentation broth, we observed that total free amino acids increased slightly with fermentation time. Lu et al. (2013) reported that some bacteria could secrete peptide enzymes and break down the protein into a variety of free amino acids, which might be the reason why total free amino acids increased slightly with fermentation time. These free amino acids served as the most important aromatic precursor and could improve the flavour quality of kombucha tea remarkably. Contrary to slight increase of amino acids, a slight decrease in caffine concentration of kombucha fermentation broth during fermentation was observed. It was speculated that the decrease might be related to the diversity of microbial community and the increasing microbial symbionts could degrade caffine to alternative carbon or nitrogen sources (Kallel et al., 2012). But it is far from obvious which microorganisms did it. Thus, more in-depth study should be carried out to prove it.

The volatile components from raw Pu-erh tea subatrates itself were primary flavour substances of kombucha tea broth at the initial fermentation stage (about 0-3 d). These constituents belonged to alcohols, aldehydes, ketones, acids, esters and benzenoids, among which linalool, benzeneacetaldehyde, heptaldehyde and 2,4-dimethyl benzaldehyde were predominant compounds providing kombucha fermentation broth a fruit aroma. However, the aromatic components reduced gradually and some of them even disappeared with the increasing microbial symbionts throughout the fermentation time.

It was considered that secondary metabolites produced by kombucha microorganism were primary flavour components in fermenting kombucha tea broth (Wang et al., 2015; Zhao et al., 2010; Sainz et al., 2016; Coton et al., 2017). In this present study, we identified 60 volatile flavour components including 7 different types: alcohols, aldehydes, ketones, acids, esters, benzenoids and others in fermenting kombucha tea broth. Among them, some predominant metabolites like acetic acids, phenylacetic acids, citric acids, D-glucuronic acids, succinic acids and other organic acids, which provide the products sour taste and beneficial effects, were always studied and recognized as main factors of causing an increase of total acidity and a decrease of $\mathrm{pH}$ value (Jayabalan et al., 2007;). Other metabolites such as 2,4-dimethyl benzaldehyde, methoxyacetic acid-4-hexadecane ester and 3-methyl propionic acid-2-acrylic este, which probably provide fermentation broth a fruit aroma, were seldom revealed in kombucha tea broth. Totally, 35 unconventional constitutions of above-mentioned volatile flavour components were analyzed-perhaps for the first time in fermenting kombucha tea broth, although such compounds in other microbial fermentation liquid had been detected before (Liu et al., 2008; Gobbi et al., 2013). We observed that volatile flavour substances, whether its composition or relative content, changed with fermentation process. More alcohols, aldehydes and acids were present at the initial to mid-fermentation stages, but the greatest variety was acids. And acetic acids and esters also increased gradually at the mid- to late-fermentation stages. It was thought that there might be many biochemical reactions, mainly including the formation of alcohols and adehydes, and the transition from alcohols and adehydes to acids and esters, under the powerful drive of kombucha microorganisms during kombucha fermentation. 
A great deal of researches showed that Kombucha microorganisms play an important role in the formation of flavour quality during kombucha fermentation (Chakravorty et al., 2016; Lin et al., 2015). In this paper, we separated and identified 8 different microbial communities in kombucha fermentation broth during kombucha fermentation. These microbial communities belonged to yeasts and bacteria. Similarly the previous studies, more Gluconacetobacter (Gluconacetobacter saccharivorans, Gluconacetobacter sp. and Gluconacetobacter europaeus) and less yeasts (only including Saccharomyces cerevisiae and Arxula adeninivorans) had been found in kombucha fermentation broth (Zhao et al., 2010; Coton et al., 2017). Here, they facilitated the kombucha fermentation to form unique flavour quality of kombucha with acetic acid-producing bacteria (including Acetobacter sp. and Acetobacter aceti). Bacteria and yeasts grew exponentially up to 5-7 days (total count of bacteria increased from $0 \mathrm{~d}$ to $5 \mathrm{~d}$; yeasts from $0 \mathrm{~d}$ to $7 \mathrm{~d}$ ), but after that bacteria and yeasts population began to decrease. Perhaps this was due to nutrients limitation or adverse condition with lower $\mathrm{pH}$ value. It was found that there was a positive correlation between such dynamic changes of bacteria and yeasts populations and the formation of flavour components especially alcohols, aldehydes,acids and esters. Therefore, we speculated that yeasts would have the priority to use sucrose to hydrolyze sucrose into glucose and also take advantage of various carbohydrates in fermentation substrates to generate various aldehydes and alcohols $(16$ cacohols were detected in this study, but 7 alcohols like 3,5-dimethyl cyclohexanol were new metabolites produced by kombucha microorganisms). Gluconacetobacter and acetic bacteria would use the glucose and other metabolites produced by yeasts to generate organic acids, which completed the alcohol-acid conversion ane led to the formation of few esters. Of course, metabolism mechanism of flavour components participated by microorganisms in kombucha fermentation broth shall be further studied in the future.

The physical and chemical conditions, such as $\mathrm{pH}$ value, and total acidity in kombucha fermentation broth, were direct evidence of the growth of different microorganisms present and the accumulation of flavour metabolic products. In this study, it was interesting that the growth of bacteria and yeasts was not absolutely consistent with the decrease rate of $\mathrm{pH}$ value and the increase rate of total acidity. Probably, there was a more direct interrelation between $\mathrm{pH}$ value, total acidity and acids like monoacids, binary acids. Kombucha microorganism is a complex microbial ecosystem and its composition changes with original place and fermentation process. During fermentation the co-relations between microorganisms are especially complex and uncertain; hence, more investigations about chemical constituents in fermenting kombucha tea broth are necessary to reveal the formation mechanism kombucha tea quality.

\section{CONCLUSION}

In the present study, main taste components including tea polyphenols, free amino acids, catechins and caffine were analyzed during kombucha fermentation. Generally, the concentration of tea polyphenols and free amino acids firstly increased at the initial stage and then decreased till the end of fermentation. Total catechins and caffine in kombucha fermentation broth were degraded progressively with fermentation time.

Dynamic changes in volatile flavour components were investigated during kombucha fermentation. 60 volatile flavour components could be divided into 7 different types: alcohols, aldehydes, ketones, acids, esters, benzenoids and others, among which predominant flavour components were acids, aldehydes, alcohols and esters such as acetic acid and 5-methyl-2-piperazinyl methanol. These were mainly produced after 5 days fermentation.

Yeasts and bacteria are the most important driving forces in the production of flavour components during kombucha fermentation. We separated and identified 8 main microbial communities in kombucha fermentation broth during kombucha fermentation: 2 species belonged to yeasts, i.e., Saccharomyces cerevisiae and Arxula adeninivorans and 6 belonged to bacteria: Gluconacetobacter saccharivorans, Acetobacter sp., Gluconacetobacter sp., Gluconacetobacter europaeus, Acetobacter aceti and Lactobacillus fermentum. Dynamic changes of bacteria and yeasts populations were consistent with variation of flavour components types and its contents.

\section{ACKNOWLEDGMENTS}

This work was supported by Science and Technology planned project from Hubei Provincial Committee of Education (Q20121208) and Development fund of Yangtze University (GRANT 801190010121).

\section{REFERENCES}

Banerjee, D., S.A. Hassarajani, B. Maity, G. Narayan, S. K. Bandyopadhya and S. Chattopadhyay. 2010. Comparative healing property of kombucha tea and black tea against indomethacin induced gastric ulceration in mice: Possible mechanism of action. Food Funct. 1(3): 284-293.

Blanc, P.J. 1996. Characterization of the tea fungus metabolites. Biotechnol. Lett. 18(2): 139-142.

Chakravorty, S., S. Bhattacharya, A. Chatzinotas, W. Chakezborty, D. Bhattacharya and R. Gachhui. 2016. Kombucha tea fermentation: Microbial and biochemical dynamics. Int. J. Food Microbiol. 220(2): 63-72.

Chen, C and B. Y. Liu. 2000. Changes in major components of tea fungus metabolites during prolonged fermentational. J. Appl. 
Microbiol. 89(5): 834-839.

Coton, M., A. Pawtowski, B. Taminiau, G. Vurgaud and F. Deniel. 2017. Unraveling microbial ecology of industrial-scale kombucha fermentations by metabarcoding and culture-based methods. Fems. Microbiol. Ecol. 93(5): Doi: 10.1093/femsec/fix048.

Dipti, P., B. Yogesh, A. K. Kain, T. Pauline, B. Anju, M. Sairam, S. S. Mongia, G. I. Kumar and W. Selvamurthy. 2003. Lead induced oxidative stress: Beneficial effects of kombucha tea. Biomed. Environ. Sci. 16(3): 276-282.

Gobbi, M., F. Comitini, P. Domizio, C. Romani, L. Lencioni, I. Mannazzu and M. Ciani. 2013. Lachancea thermotolerans and Saccharomyces cerevisiae in simultaneous and sequential cofermentation: A strategy to enhance acidity and improve the overall quality of wine. Food Microbiol. 33(2): 271-281.

Jayabalan, R., S. Marimuthu and K. Swaminathan. 2007. Changes in content of organic acids and tea polyphenols during Kombucha tea fermentation. Food Chem. 102 (1): 392-398.

Jayabalan, R., P. N. Chen, Y. S. Hsieh, K. Prabhakaran, P. Pitchai, S. Marimuthu, P. Thangaraj, K. Swaminathan and S. E. Yun. 2011. Effect of solvent fractions of Kombucha tea on viability and invasiveness of cancer cells-characterization of dimethyl 2-(2-hydroxy-2-methoxypropylidine) malonate and vitexin. Indian J. Biotechnol. 10(1): 75-82.

Jayabalan, R., R. V. Malba`sa, E. S. Lon`car, J. S. Vitas and M. Sathishkumar. 2014. A review on kombucha tea--microbiology,composition, fermentation, beneficial effects, toxicity, and tea fungus. Compr. Rev. Food Sci F. 13(4): 538-550.

Jiang, L.W., D. H. Liu, L. Y. Liao and D. F. Tang. 2007. Study on changes in major components during tea fungus fermentation. Food Sci. 28(3): 238-240.

Kallel, L., V. Desseaux, M. Hamdi, P. Stocher and E. H. Ajandouz. 2012. Insights into the fermentation biochemistry of kombucha teas and potential impacts of kombucha drinking on starch digestion. Food Res. Int. 49(1): 226-232.

Lin, J., X. Y. Ye, Z. L. Cao, F. Y. Xie and M. A. Xu. 2015. Isolation of microbes from kombucha and kombucha fermentation with pure culture combination. J. Chin. Inst. Food Sci. Tech. 15(2): 39-48.

Liu, X.Y., H. Xu, H. Cao and X. L. Sun. 2008. GC-MS Analysis of five individual and mixture bacterium fermentation liquor metabolic product. Food Res. Dev. 29(2): 133-136.

Lu, W. G., Z. Wen, H. Li, D. Yuan, J. Li, H. Zhang, Z. Huang, S. Cui and W. Du. 2013. Identification of the quantitative trait loci (QTL) underlying water soluble protein content in soybean. Theor. Appl. Genet. 126(2): 425-433.

Marsh, A.J., O. OSullivan, C. Hill, R. P. Ross and P. D. Cotter. 2014. Sequence-based analysis of the bacteria and fungal compositions of multiple kombucha (tea fungus) samples. Food Microbiol. 38: 171-178.

Sainz, F., D. Navarro., E. Mateo, M. J. Torija and A. Mas. 2016. Comparison of D-gluconic acid roduction in selected strains of acetic bacteria. Int. J. Food Microbiol. 222: 40-47.

Screeramulu, G., Y. Zhu and W. Knol. 2000. Kombucha fermentation and its antimicrobial activity J. Agr. Food Chem. 48(6): 2589-94.

Wang, G. Z., J. Lin, X. Y. Ye, Z. L. Cao and Y. C. Li. 2015. The antimicrobial and antioxidant activities of Kombucha. J. Chin. Inst. Food Sci. Tech. 15(9): 173-179.

Xu, X., M. Yan and Y. Zhu. 2005. Influence of fungal fermentation on the development of volatile compounds in the puer tea manufacturing process. Chemosphere. 5(4): 382-386.

Yang, Z.W., B. P. Ji, F. Zhou, B. Li, Y. Luo, L. Yang and T. Li. 2009. Hypocholesterolaemic and antioxidant effects of kombucha tea in high-cholesterol fed mice. J. Sci. Food Agr. 89(1): 150-156.

Zhao, Z. J., H. R. Tong, L. Zhou and Q. J. Liu. 2010. Variations in major components of pu' er tea infusion during fermentation by kombucha culture. J. Food Sci. 31(1): 79-83. 